\title{
Spatial Patterns of Dissipative Polariton Solitons in Semiconductor Microcavities
}

\author{
J. K. Chana, ${ }^{1}$ M. Sich, ${ }^{1,}{ }^{*}$ F. Fras, ${ }^{1,2}$ A. V. Gorbach, ${ }^{3}$ D. V. Skryabin,${ }^{3,4}$ E. Cancellieri, ${ }^{1}$ E. A. Cerda-Méndez, ${ }^{5}$ \\ K. Biermann, ${ }^{5}$ R. Hey, ${ }^{5}$ P. V. Santos, ${ }^{5}$ M. S. Skolnick, ${ }^{1}$ and D. N. Krizhanovskii ${ }^{1, \dagger}$ \\ ${ }^{1}$ Department of Physics and Astronomy, The University of Sheffield, Sheffield S3 7RH, United Kingdom \\ ${ }^{2} I P C M S$ UMR 7504, CNRS and Université de Strasbourg, Strasbourg 67084, France \\ ${ }^{3}$ Department of Physics, University of Bath, Bath BA2 7AY, United Kingdom \\ ${ }^{4}$ ITMO University, Kronverksky Avenue 49, St. Petersburg 197101, Russian Federation \\ ${ }^{5}$ Paul-Drude-Institut für Festkörperelektronik, Berlin 10117, Germany
}

(Received 1 December 2014; revised manuscript received 23 July 2015; published 15 December 2015)

\begin{abstract}
We report propagating bound microcavity polariton soliton arrays consisting of multipeak structures either along $(x)$ or perpendicular $(y)$ to the direction of propagation. Soliton arrays of up to five solitons are observed, with the number of solitons controlled by the size and power of the triggering laser pulse. The breakup along the $x$ direction occurs when the effective area of the trigger pulse exceeds the characteristic soliton size determined by polariton-polariton interactions. Narrowing of soliton emission in energymomentum space indicates phase locking between adjacent solitons, consistent with numerical modeling which predicts stable multihump soliton solutions. In the $y$ direction, the breakup originates from inhomogeneity across the wave front in the transverse direction which develops into a stable array only in the solitonic regime via phase-dependent interactions of propagating fronts.
\end{abstract}

DOI: 10.1103/PhysRevLett.115.256401

PACS numbers: 71.36.+c, 05.45.Yv, 78.67.Pt

Solitons occur when dispersive spreading of wave packets is compensated through nonlinear interactions. They have been observed in numerous systems including nonlinear crystals and optical fibers [1,2], atomic BoseEinstein condensates [3], and natural processes including signal propagation in neurons [4], cloud formation [5], and large-amplitude waves [6,7]. In many aspects, solitons behave like artificial particles. They may repel or attract depending on their relative phase as was shown in optical fibers [8] and cold atom systems [9]. Multisoliton complexes can form when localized initial perturbations split into multiple peaks $[10,11]$. Furthermore, solitons can selforganize into stable patterns with an equilibrium spacing [12] or can scatter in a collision [13].

Recently, polaritons, hybrid light-matter particles forming in the strong coupling regime in semiconductor microcavities [14,15], have been shown to exhibit many interesting nonlinear hydrodynamic phenomena such as superfluidity [16] and integer [17] and half vortices [18]. Microcavity polaritons are an open system far from equilibrium. Bright polariton solitons have been observed [19], which exist when an external pump fully compensates photonic losses and the decay of the excitonic coherence and are therefore termed dissipative. Polariton solitons can be manipulated on a picosecond time scale, which is promising for the development of miniature polaritonic

Published by the American Physical Society under the terms of the Creative Commons Attribution 3.0 License. Further distribution of this work must maintain attribution to the author $(s)$ and the published article's title, journal citation, and DOI. circuits and logic gates [20]. Dark soliton trains in a 1D conservative microcavity system (no pump) were recently predicted theoretically [21], whereas dissipative polariton soliton patterns in microcavities remain unexplored.

Semiconductor optical resonators are prone to growth defects and imperfections [22], and, in contrast to atomic condensates [11], the observation of multiple solitons in such systems is a challenging task, even in well-studied systems such as vertical-cavity surface-emitting lasers (VCSELs) [23,24]. In contrast to solitons in VCSELs, microcavity polariton solitons are excited at high momenta. Combined with large energy blueshifts due to the giant optical nonlinearity, this makes polariton solitons less sensitive to photonic disorder, enabling our observation of stable multisoliton patterns.

Here we demonstrate the formation of dissipative polariton soliton patterns. The interplay between bistability of the external pump field, polariton-polariton scattering, and polariton negative effective mass along the propagation direction enables the formation of $x$-soliton arrays, i.e., bound solitons following one another in space and time. The $k$-space emission patterns observed indicate phase locking between adjacent solitons. Up to five stable bound solitons are observed in agreement with numerical modeling predicting stable multihump solutions. Soliton separations in a time of $\approx 10 \mathrm{ps}$ are observed in the $x$ arrays, potentially promising for the development of polaritonic devices operating at $\approx 100 \mathrm{Gbit} / \mathrm{s}$. We also observe stable $y$-soliton arrays formed perpendicular to the propagation direction, arising from changes of the front velocity across the propagating beam profile. 

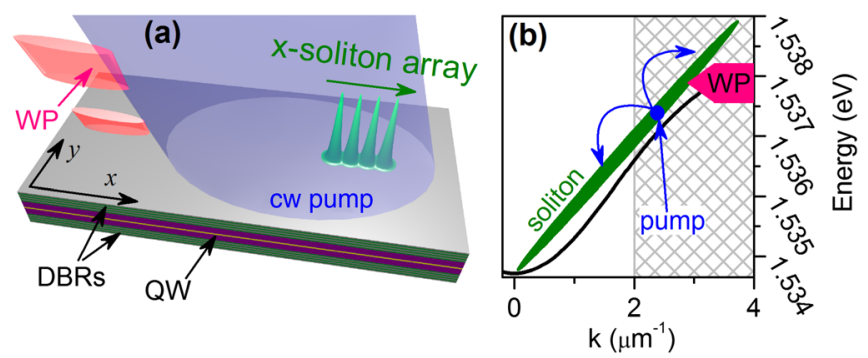

FIG. 1 (color online). (a) Schematic diagram of the experiment. A large area of the sample is quasiresonantly illuminated with a cW pump. A WP (red) triggers the soliton pattern formation. (b) Polariton dispersion (solid black curve) of the lower polariton branch. The schematic location of the pump and WP in energymomentum space are shown; the shaded area above $k>2 \mu \mathrm{m}^{-1}$ is blocked in the detection path to avoid saturation of the detectors by the reflected pump and WP beams [25].

Results.-In our work, we used the sample described in Supplemental Material [25]. The basics of the soliton excitation are explained in Fig. 1. The writing pulse (WP) which triggers soliton formation is chosen to be elongated along [Fig. 1(a)] or perpendicular to [Fig. 4(a)] the direction of soliton propagation. We name the resulting patterns in the two cases $x$-soliton arrays and $y$-soliton arrays, respectively, for ease of discussion. $x$-soliton arrays are bound solitons moving one after another, while in the case of $y$-soliton arrays we observe multipeak patterns along the transverse direction moving as a single front. All experiments reported here were performed in a single polarization configuration with the pump and WP cocircularly polarized to minimize polarization cross talk [28].

$x$-soliton arrays.-Bright polariton solitons are supported by a continuous wave (cw) $300 \mathrm{~mW}$ pump resonant with the lower polariton branch at high momentum $k \approx 2.1 \mu \mathrm{m}^{-1}$. The pump power is tuned into the bistability domain; the soliton can be considered as a local switch from the lower to the upper state on the bistability curve. Parametric polariton-polariton scattering from the pump populates soliton harmonics over a broad range of $k$ vectors. In order to excite $x$-soliton arrays, we apply an elongated WP at the edge of the Gaussian $\mathrm{cw}$ pump spot [Fig. 1(a)], i.e., within $\approx 20 \mu \mathrm{m}$ from the region where the pump exhibits bistable behavior. The WP is quasiresonant with the lower polariton branch and its transverse momentum $k_{\mathrm{wp}}$ and energy $\hbar \omega_{\mathrm{wp}}$ are close to that of the pump: $k_{\mathrm{wp}} \approx 2.5 \pm 0.1 \mu \mathrm{m}^{-1}, \hbar \omega_{\mathrm{wp}} \approx 1.5367 \pm 0.0002 \mathrm{eV}$. Full widths at half maxima (FWHM) of the Gaussian WP are $30 \mu \mathrm{m}$ by $5 \mu \mathrm{m}$ along directions $x$ (soliton propagation direction) and $y$ (transverse direction), respectively, and the pulse duration is 5 ps FWHM (through spectral filtering). See the Supplemental Material [25] for more details about the experimental setup.

Polariton solitons are triggered at WP power densities above a certain threshold [19]. At low WP powers, only a small region in the center of the Gaussian WP is expected to excite a single soliton whose size is determined by the polariton-polariton interactions and the cavity parameters [19]. At higher WP powers, the area across the injected polariton wave packet where solitons may be excited becomes larger than the soliton size, so multiple bound solitons are triggered. This is similar to atomic condensates, which break up into soliton trains when the condensate size is larger than the condensate healing length [11]. Figures 2(a)-2(e) show spatiotemporal profiles of solitons for different WP powers. The number of created solitons increases with the WP power. At first, at a WP power of $0.3 \mathrm{~mW}$, only one soliton is excited [Fig. 2(a)], whereas at $0.5 \mathrm{~mW}$ two parallel soliton traces are observed [Fig. 2(b)]. Notably, as the WP power increases, the spacing between solitons gradually increases, and more soliton traces appear at higher WP power [Figs. 2(d) and 2(e)]. Profiles in time of the five-peak and three-peak structures are shown in Figs. 2(g) and 2(h) with the FWHM of a single peak ranging from 4 to $7 \mathrm{ps}$ (corresponding to $\approx 8-14 \mu \mathrm{m}$ along the $x$ axis). All solitons travel at the same speed, indicating the formation of stable soliton patterns. Figure 2(f) shows
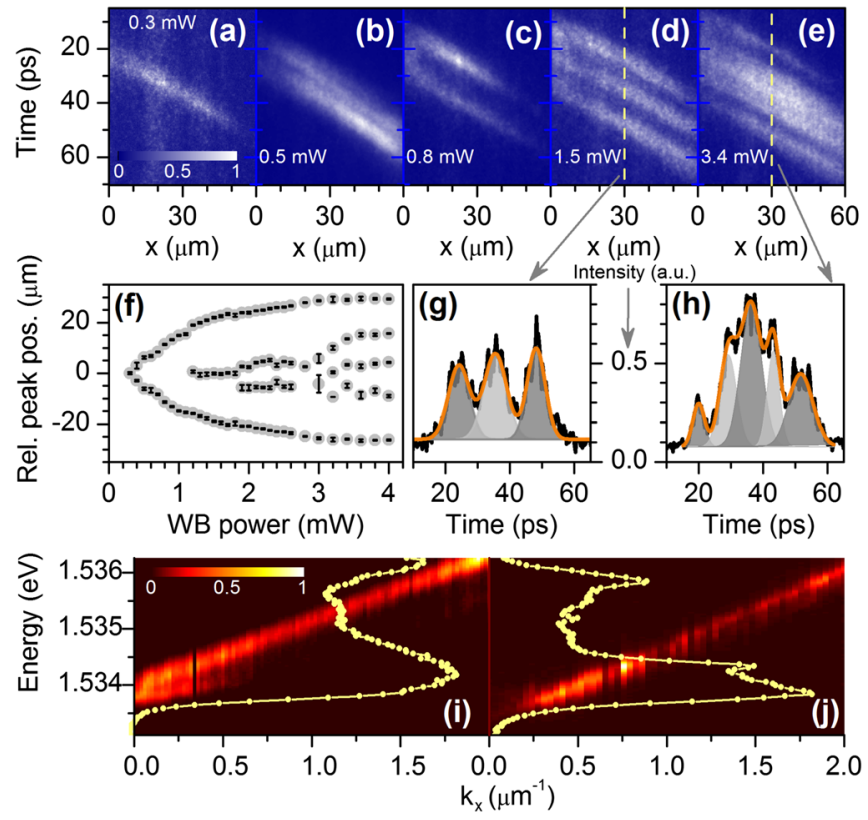

FIG. 2 (color online). (a)-(e) Emission intensities of a single soliton (a) and $x$-soliton arrays (b)-(e) for different WP powers recorded versus time and position $x ; t \approx 0$ is the WP arrival time within an error of 5 ps. The pump beam is centered around $x=30 \mu \mathrm{m}$. (f) Average relative positions of soliton traces as a function of WP power. $(\mathrm{g}),(\mathrm{h})$ Intensity versus time profiles at $x=30 \mu \mathrm{m}$ of the three-peak and five-peak $x$-soliton arrays in (d), (e), where the average soliton duration is $\approx 6$ ps and the velocity is $\approx 2.2 \mu \mathrm{m} /$ ps. (i),(j) Intensity of a single soliton and four-peak $x$-soliton array in $E-k_{x}$ space at $\approx 30$ ps taken at $k_{y}=0$ within $\Delta k_{y}=0.1 \mu \mathrm{m}^{-1}$; the yellow lines show the total intensity distribution of the emission versus energy. 
the WP power dependence of the positions of soliton peaks relative to the middle of the corresponding soliton patterns [29].

A difference between solitons and dispersive wave packets is that the soliton dispersion is expected to be linear due to polariton-polariton interactions [19]. Figures 2(i) and 2(j) [30] display the experimental measurements of the energy versus momentum distribution $I\left(E, k_{x}\right)$ for a single soliton and a four-peak $x$-soliton array (the soliton time profiles are shown in Ref. [25]). In both cases, the spectra exhibit linear dispersion of the soliton emission at all $k$ vectors down to zero in strong contrast to the dispersion of the lower polariton branch, which is parabolic at $k \approx 0$ [Fig. 1(b)]. The yellow lines in Figs. 2(i) and $2(\mathrm{j})$ show the total intensity distribution of the soliton emission versus energy $\left[I(E)=\int_{0}^{2} \mu \mathrm{m}^{-1} I\left(E, k_{x}\right) d k_{x}\right]$ with two maxima at around 1.534 and $1.536 \mathrm{eV}$. These peaks become narrower and more pronounced with an increasing number of soliton peaks, which has been predicted theoretically [31]. This narrowing in energy-momentum space is an indication of phase locking of spectral harmonics of adjacent solitons leading to the onset of spatiotemporal coherence across the soliton array. The phase locking occurs because the tails of solitons overlap in real space. Hence, polaritons residing at different $k$ vectors in one soliton stimulate parametric scattering to spectral harmonics of adjacent solitons.

We have further confirmed the solitonic nature of the patterns by verifying that similar results are obtained for different values of $k_{\mathrm{wp}}$ and energies $E_{\mathrm{wp}}$. The exact profile of the soliton train may change with $k_{\text {wp }}$ and $E_{\text {wp }}$, but the dispersions remain linear with a pronounced narrowing of the emission in energy-momentum space with an increasing number of solitons. The soliton spectrum is broad and is populated by parametric scattering from the pump state which may be triggered to form a soliton by a WP anywhere on the soliton dispersion.

For numerical investigation of the system, we used Gross-Pitaevskii equations describing coupled TE and TM cavity modes interacting with the spin-dependent polariton field [19] and looked for quasicircularly polarized soliton solutions moving in the direction of the pump momentum (i.e., along the $x$ axis) [19]. The model is described in Supplemental Material [25]. Previously $[19,28]$, it has proved to include all the important features necessary to numerically reproduce the experimental conditions. The soliton existence range in terms of the pump intensity, $\left|E_{p}\right|^{2}$, is well approximated by the bistability interval of the intracavity field [19].

We have computed the bifurcation diagram showing a sequence of stable multipeak solitons [Fig. 3(a)]. The diagram shows that, for every given pump intensity (horizontal axis) within the bistability range, stable soliton solutions with different numbers of peaks can be excited (vertical axis). An example of a five-hump soliton is shown
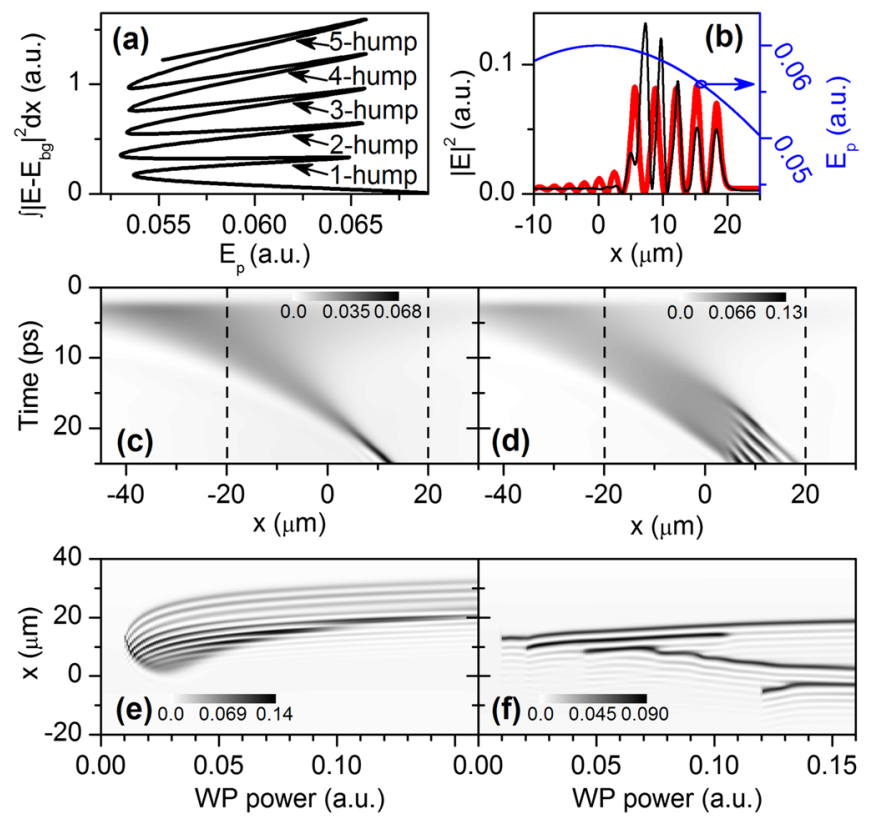

FIG. 3 (color online). (a) Snakelike bifurcation diagram for multipeak solitons in the case of an infinite flat pump. $E(x)$ is the electric field amplitude, $E_{b g}$ is the amplitude of the homogeneous solution (in the tails of the soliton), and $E_{p}$ is the pump amplitude. The homogeneous solution is bistable in the range $0.052<E_{p}<0.070$. (b) The red line is an example of the exact five-peak $x$-soliton array solution found for an infinite flat pump with amplitude $E_{p}=0.06$; the black line is the five-peak $x$ soliton array generated in the numerical modeling reproducing the experimental conditions of the Gaussian pump profile; the blue line shows the Gaussian profile of the pump field. (c), (d) Spatiotemporal dynamics (gray scale is $|E|^{2}$ ) resulting in the formation of the single-peak (c) and four-peak (d) $x$-soliton array. The pump field has a Gaussian profile with amplitude $E_{p}=0.06$, and the corresponding boundaries of the bistability region [as in (a)] are indicated by vertical dashed lines. The WP amplitude is $E_{\text {wp }}=0.1$ (c) and $E_{\text {wp }}=0.12$ (d). (e),(f) Gray-scale profiles of multipeak structures recorded in numerical simulations after 25 ps as a function of the WP power. In (e) $k_{\mathrm{wp}}=k_{p}$, while in (f) the WP momentum is offset by $3^{\circ}\left(\Delta k \approx 0.4 \mu \mathrm{m}^{-1}\right)$.

in Fig. 3(b). In our time-dependent modeling, we assumed a Gaussian profile of the WP. With an increasing WP intensity, there is an increasing number of peaks in stable multipeak soliton solutions [Figs. 3(c) and 3(d)].

In practice, the profile of the pump is Gaussian, and therefore conditions for the soliton formation are met only within a certain radius around the pump center, where the pump intensity is large enough to bring the cavity into the bistable regime. In Fig. 3(b), we compare profiles found in the case of an infinite flat pump with those moving through the Gaussian pump. One can see that in both cases the peak widths and peak spacings are closely matched. The peak amplitudes, however, differ, as the latter structure does not spend sufficient time inside the bistability range to fully stabilize itself. 
Figure 3(f) shows how the number and relative position of peaks vary with the WP power for slightly offset momenta of the pump and WP beams. Figure 3(f) is in good qualitative agreement with the experimental observations in Fig. 2(f) (see Supplemental Material [25] for the effects of $k$-space filtering). With increasing WP power, there is an increase in both the number of peaks and the spacings between the solitons up to 10-20 $\mu \mathrm{m}$. In numerical simulations minimizing the difference between $k_{\mathrm{wp}}$ and $k_{p}$ (preferably to zero) facilitates the generation of a regular pattern of peaks, while in the experiment $k_{\mathrm{wp}}-k_{p} \approx$ $0.4 \mu \mathrm{m}^{-1}$ due to physical constraints of the setup.

The solitons are formed by scattering polaritons from the pump state to populate the soliton spectrum. The characteristic time of polariton scattering is approximately given by $h /(g N)$, where $g$ is the interaction constant and $N$ is the density (then $g N$ is the blueshift). For a blueshift of $0.3 \mathrm{meV}$, about $13 \mathrm{ps}$ are needed to fully populate the spectrum and form a soliton. In numerical modeling, for a Gaussian pump spot where $k_{\mathrm{wp}} \approx k_{p}$, solitons will form only after the polaritons injected by the WP have propagated a certain distance through the pump bistability region [Figs. 3(c) and 3(d)]. By contrast, for $k_{\mathrm{wp}}>k_{p}$, interactions between the polariton populations injected by the pump and WP facilitate breakup of the WP polaritons into localized wave packets before or at the boundary of the pump bistability area $x \approx 0$ [see Figs. 2(b) and 2(e) and Ref. [25] for further discussion].

$y$-soliton arrays. - We note that localization along the $x$ direction occurs due to the interplay between the negative effective mass and repulsive interactions. However, we observe that solitons are also localized along the $y$ direction due to the interaction of propagating fronts combined with the phase-dependent parametric nonlinearity. Qualitatively, this localization may be explained by the effective potential, which is created in the pump state due to pump depletion by parametric scattering [31].

We have experimentally investigated how localization along the $y$ direction affects pattern formation. We elongate the WP along the $y$ direction, so it is $\approx 7 \mu \mathrm{m}$ along the $x$ axis and $\approx 30 \mu \mathrm{m}$ along the $y$ axis. In this case, we observe breaking of the initially smooth beam profile into a $y$-soliton array; see Fig. 4. As with the $x$-soliton arrays, the formation of the patterns occurs only in the soliton regime. The same experiment when the pump power is below the bistability regime never leads to the formation of stable patterns but to a dispersive wave packet.

The number of peaks created depends, as for the $x$-soliton arrays, on the effective spot size of the WP. Figures 4(b)-4(e) show real-space images of the emerging structures for different WP powers. Peaks are spaced by $\approx 8 \mu \mathrm{m}$, are of the size (FWHM) of $\approx 7 \mu \mathrm{m}$ [see Fig. 4(f)], and travel along the $x$ direction within the pump bistability area.

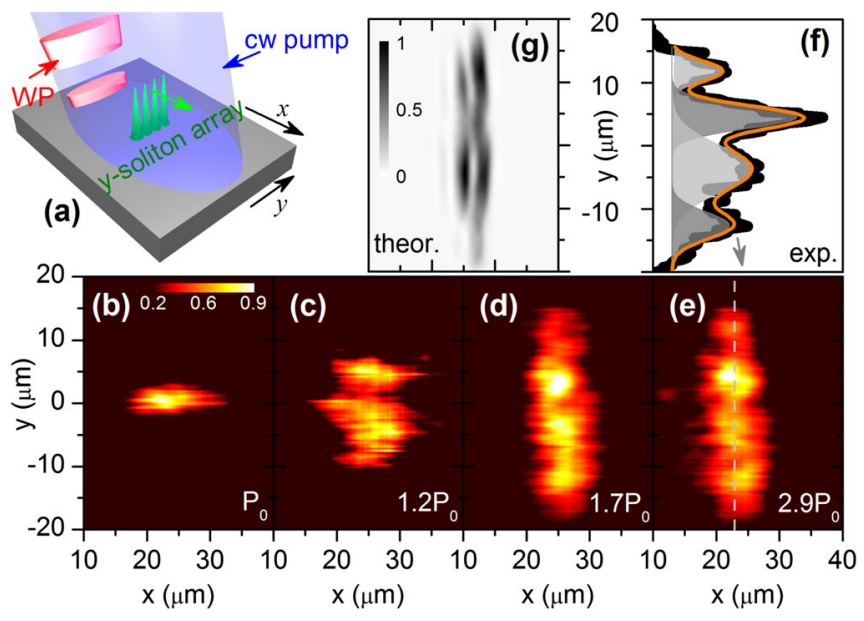

FIG. 4 (color online). (a) Experimental setup with the WP elongated along the $y$ axis. (b)-(e) 2D images of one- to four-peak arrays obtained for different WP powers, $P_{0}=230 \mu \mathrm{W}$, at time $t=20 \mathrm{ps}$ after the WP arrival. (f) Profile of a four-peak array along the $y$ axis. (g) Numerically modeled transverse distribution of the intracavity optical intensity after 15 ps of propagation under the conditions when the WP is elongated along the $y$ coordinate (as in the experimental results) and a $3^{\circ}$ angle between the WP and pump momenta. As in the experiment, this figure is averaged over the phase of the WP.

Similar to the $x$-soliton array, increasing the power leads to the creation of additional peaks on the "sides" of the WP. It is important to note that the location of solitons triggered in such a way does not depend on the position on the sample: moving the sample by $10 \mu \mathrm{m}$ relative to the laboratory frame does not significantly influence the shape of the array. At the same time, moving the WP along the $y$ axis relative to the pump and sample, which are fixed in the laboratory frame, leads to the respective shift of the soliton array trajectory [25].

In order to explain the mechanism of pattern formation along the $y$ direction, we first take a single-hump onedimensional soliton localized along the $x$ direction and infinitely extended along $y$ and performed its linear stability analysis. This demonstrates that it is stable with respect to any transverse instabilities, which could lead to filamentation along the $y$ direction. If, however, we limit the extent of the soliton stripe by imposing a Gaussian profile in the $y$ direction, then the change in the curvature of the soliton front leads to changes of the front velocity across the beam profile. This in turn leads to the breakup of the wave front and the formation of $y$-soliton arrays, as in the experiment. The latter process develops faster and is more pronounced if a small angle between the pump and WP beams in the $y$ plane is introduced [see Fig. 4(g)]. In this case, the interference between the WP and pump beams creates an initial modulation of polariton density across the $y$ direction, which assists the breakup of the polariton wave packet into a $y$-soliton array. 
Conclusions. - In the present study, we have shown the existence and evolution of dissipative multisoliton structures. $x$-soliton arrays and $y$-soliton arrays can be triggered by elongating the WP along the $x$ and $y$ directions, respectively. Increasing the WP effective size (by changing its power) leads to the creation of larger structures with more peaks; however, the physical mechanisms underlying breakup in the $x$ and $y$ directions are very different. $x$ soliton arrays can be excited across a broad range of initial conditions. This feature is typical for dissipative systems, where the soliton shape and energy are predetermined by the system parameters rather than the initial conditions.

We acknowledge support from the Leverhulme Trust Grant No. PRG-2013-339, EPSRC Grant No. EP/J007544/ 1, and ERC Advanced Grant No. EXCIPOL 320570. D. V. S. acknowledges support from the Government of the Russian Federation (Grant No. 074-U01) through the ITMO visiting professorship scheme. D. V.S. and A. V. G. acknowledge support from the EU network project LIMACONA (Project No. 612600) and from the Leverhulme Trust Grant No. RPG-2012-481.

*m.sich@sheffield.ac.uk

†.krizhanovskii@sheffield.ac.uk

[1] Y. Kivshar and G. Agrawal, Optical Solitons: From Fibers to Photonic Crystals (Academic, New York, 2003).

[2] T. Ackemann, W. J. Firth, and G. L. Oppo, in Advances in Atomic, Molecular and Optical Physics, edited by E. Arimondo, P. R. Berman, and C. C. Lin (Academic, New York, 2009), Vol. 57, pp. 323-421.

[3] L. Khaykovich, F. Schreck, G. Ferrari, T. Bourdel, J. Cubizolles, L. D. Carr, Y. Castin, and C. Salomon, Science 296, 1290 (2002).

[4] S. S. L. Andersen, A. D. Jackson, and T. Heimburg, Prog. Neurobiol. 88, 104 (2009).

[5] P. G. Baines, Topographic Effects in Stratified Flows, Cambridge Monographs on Mechanics Series (Cambridge University Press, Cambridge, England, 1998).

[6] J. S. Russell, Report on Waves, in Report of the Fourteenth Meeting of the British Association for the Advancement of Science, York, 1844 (John Murray, London, 1845), Vol. 14, pp. 311-390, Plates XLVII-LVII.

[7] J. R. Apel, L. A. Ostrovsky, Y. A. Stepanyants, and J. F. Lynch, technical report, Woods Hole Oceanographic Institution, 2006.

[8] J. S. Aitchison, A. M. Weiner, Y. Silberberg, D. E. Leaird, M. K. Oliver, J. L. Jackel, and P. W. E. Smith, Opt. Lett. 16, 15 (1991).

[9] J. Nguyen, P. Dyke, D. Luo, B. Malomed, and R. Hulet, Nat. Phys. 10, 918 (2014).

[10] G. Slekys, K. Staliunas, and C. O. Weiss, Opt. Commun. 149, 113 (1998).

[11] K. E. Strecker, G. B. Partridge, A. G. Truscott, and R. G. Hulet, Nature (London) 417, 150 (2002).

[12] B. Schäpers, M. Feldmann, T. Ackemann, and W. Lange, Phys. Rev. Lett. 85, 748 (2000).
[13] A. J. Scroggie, J. M. McSloy, and W. J. Firth, Phys. Rev. E 66, 036607 (2002).

[14] A. Kavokin, J. J. Baumberg, G. Malpuech, and F. P. Laussy, Microcavities, Semiconductor Science and Technology (Oxford University, New York, 2007).

[15] D. Sanvitto and V. B. Timofeev, Exciton Polaritons in Microcavities: New Frontiers, Solid State Sciences Vol. 172 (Springer, New York, 2012).

[16] A. Amo, J. Lefrere, S. Pigeon, C. Adrados, C. Ciuti, I. Carusotto, R. Houdre, E. Giacobina, and A. Bramati, Nat. Phys. 5, 805 (2009).

[17] G. Nardin, G. Grosso, Y. Léger, B. Pietka, F. MorierGenoud, and B. Deveaud-Plédran, Nat. Phys. 7, 635 (2011).

[18] K. G. Lagoudakis, T. Ostatnický, A. V. Kavokin, Y. G. Rubo, R. André, and B. Deveaud-Plédran, Science 326, 974 (2009).

[19] M. Sich, D. N. Krizhanovskii, M. S. Skolnick, A. V. Gorbach, R. Hartley, D. V. Skryabin, E. A. Cerda-Méndez, K. Biermann, R. Hey, and P. V. Santos, Nat. Photonics 6, 50 (2012).

[20] M. De Giorgi, D. Ballarini, E. Cancellieri, F. M. Marchetti, M. H. Szymanska, C. Tejedor, R. Cingolani, E. Giacobino, A. Bramati, G. Gigli, and D. Sanvitto, Phys. Rev. Lett. 109, 266407 (2012).

[21] F. Pinsker and H. Flayac, Phys. Rev. Lett. 112, 140405 (2014).

[22] T. Ackemann, N. Radwell, Y. Noblet, and R. Jäger, Opt. Lett. 37, 1079 (2012).

[23] V. B. Taranenko, I. Ganne, R. J. Kuszelewicz, and C. O. Weiss, Phys. Rev. A 61, 063818 (2000).

[24] S. Barland, J. R. Tredicce, M. Brambilla, L. A. Lugiato, S. Balle, M. Giudici, T. Maggipinto, L. Spinelli, G. Tissoni, T. Knudl, M. Miller, and R. Jager, Nature (London) 419, 699 (2002).

[25] See Supplemental Material at http://link.aps.org/ supplemental/10.1103/PhysRevLett.115.256401 for details of the experimental setup, numerical model, additional images of $x$-soliton arrays and $y$-soliton array stability study, which include Refs. [19,26,27].

[26] Hamamatsu Photonics K. K., Universal Streak Camera C10910 Series.

[27] O. Egorov, D. Skryabin, A. Yulin, and F. Lederer, Phys. Rev. Lett. 102, 153904 (2009).

[28] M. Sich, F. Fras, J. K. Chana, M. S. Skolnick, D. N. Krizhanovskii, A. V. Gorbach, R. Hartley, D. V. Skryabin, S. S. Gavrilov, E. A. Cerda-Mèndez, K. Biermann, R. Hey, and P. V. Santos, Phys. Rev. Lett. 112, 046403 (2014).

[29] The positions of soliton peaks in Fig. 2(f) are defined as $x=1 / T \int_{0}^{T}\left[x(t)-x_{0}(t)\right] d t$, where $x(t)$ is the trace of the soliton peak of interest; $x_{0}(t)$ is the middle between the two outer solitons $x_{1}(t), x_{2}(t)$ in each pattern-i.e., $x_{0}(t)$ fits $1 / 2\left[x_{1}(t)+x_{2}(t)\right]$ with the soliton velocity $v \approx$ $2.2 \mu \mathrm{m} / \mathrm{ps}$ - and $T$ is the propagation time of each soliton within the observation window.

[30] The soliton dispersions in Figs. 2(i) and 2(j) were measured for slightly different positions on the sample. The exciton-photon detunings were therefore different, leading to soliton dispersions shifted with respect to each other by about $0.5 \mathrm{meV}$.

[31] O. A. Egorov, A. V. Gorbach, F. Lederer, and D. V. Skryabin, Phys. Rev. Lett. 105, 073903 (2010). 